\title{
Filament Power Supply Improvement of the TRIUMF RF System
}

\author{
Amiya Kumar Mitra and Joseph J. Lu \\ TRIUMF \\ 4004 Wesbrook Mall, Vancouver, BC, Canada V6T 2 A3.
}

\section{Abstract}

The TRIUMF $r$ system, operating at a fixed frequency of $23.06 \mathrm{MHz}$, employs four push-pull power amplifiers using eight EIMAC 4CW250,000 tetrodes. The filament power supplies for the above tetrodes uses simplified 3 phase SCR control circuits to slowly ramp up the output voltage. Failure of SCR's during ramping and also during power bumps has been a source of rf downtime. A new SCR control scheme has been developed and tested which relies on the fact that the secondary output voltage from a 3 phase transformer will be $57.7 \%$ of it's rated output voltage if only two phases of the primary are connected. In this scheme, one phase is connected directly and the second phase is connected through two SCR's of which the conduction angle is controlled by a slow ramp. The third phase is initially blocked by two SCR's which are turned on fully after a fixed delay thereby limiting the surge current of the filament during start up. A greatly simplified firing circuit and smaller number of components has improved the reliability and the performance of such a power supply. Also, an alternate scheme of a power supply with series resistors in the primary of the transformer is described.

\section{INTRODUCTION}

The TRIUMF rf system [1] and [2] operates at a fixed frequency of $23.06 \mathrm{MHz}$ with a power capability of $1.8 \mathrm{MW}$. Four push-pull power amplifiers employing eight EIMAC $4 \mathrm{CW} 250,000$ tetrodes generate the required power. Since the cold resistance of the filament is much smaller than the nominal resistance (11.5 V/900 Amps), slow ramping of the oulpul vollage of the filament power supply is needed to limit the surge current to its rated value. The original power supply uses three phase transformer, full wave rectificr, choke and filter capacitor to produce the required rated dc voltage and current. It employs one SCR and one diode back to back in each phase in the primary of the transformer. It means only half of the cycle is controlled. Small unbalances among the trigger signals in three phases may trip off the $\mathrm{AC}$ circuit breaker or even damage the SCR's during the ramp up period. Many hours of beam time was lost due to the malfunction of these power supplies. Also, repairing of these power supplies was time consuming due to the fact that various voltages and time constants in the firing circuit of the SCR's had to be set precisely to obtain balanced triggering for all the SCR's. Various options for the filament power supplies were considered and a new SCR control circuit has been designed. This circuit only controls the conduction angle of one phase. The second phase is always connected and the third phase is turned on after a fixed delay, with SCR's acting as a switch. An alternate scheme using a power supply with series resistors in the primary of the transformer was used as a quick interim solution.

\section{POWER SUPPLY REQUIREMENTS}

The filament power supply for the EIMAC 4CW250,000 must fulfill the following requirements:

Rated maximum output voltage : $11.5 \mathrm{~V} \mathrm{dc}$. Rated maximum output current : 900 Amps. Maximum surge current must be limited to 1800 Amps. The maximum in-rush current of the $440 \mathrm{~V}$ ac circuit breaker should not exceed 15 Amps.

\section{SINGLE PHASE CONTROLLER}

The single phase controller uses two SCR's, SCR1 and SCR2, back to back of which the conduction angle is controlled by a ramp voltage such that in about 20 seconds $57 \%$ of the rated filament output voltage is gradually established. This is due to the fact that when only two phases of a three phase transformer are energized 0.577 of the nominal output voltage is obtained. The filament is heated under this voltage for about 20 seconds, it's resistance rises

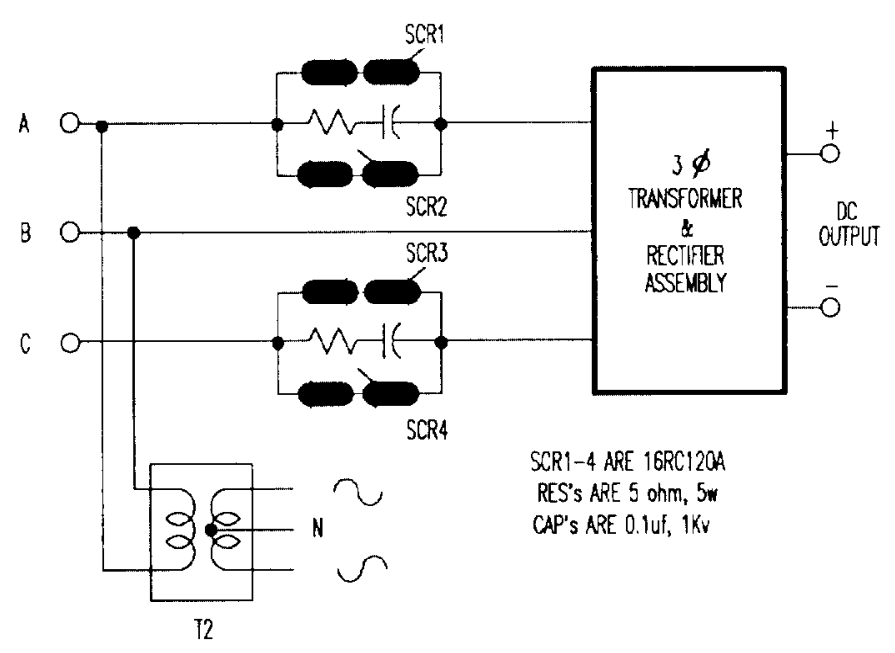

Figure 1. Basic scheme of the single phase SCR controller. 
to nearly $80 \%$ of it's rated value. The third phase is then fully turned on by switching on SCR3 and SCR4 to achieve the full rated output voltage and the surge current is limited to 1.25 times the nominal filament current of 670 Amps.

Figure 1 shows the basic scheme of the single phase SCR controlled circuit. $\mathrm{T} 2$ is a 480 to 30 Volt transformer which provides 1): the phase reference for the control circuit, and $2)$ : the ac source for the controller's dc supplies.

\section{Circuit Description}

Figure 2 is the block diagram of the system which consists mainly of a de supply for the controller, a ramp generator, a $20 \mathrm{kHz}$ oscillator, firing angle control circuit, three trigger drivers and a counter to provide 20 or 40 seconds delay. The system is constructed on two circuit boards. The ramping board consists of the dc supply, the ramp generator and the $20 \mathrm{kHz}$ oscillator and the firing board consists of the rest of the building blocks.

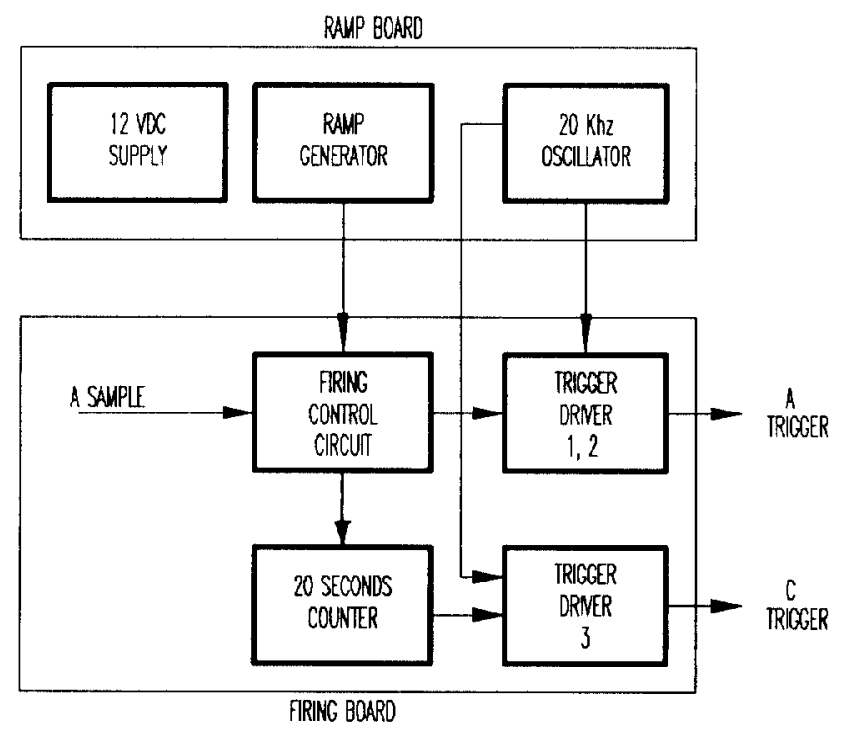

Figure 2. Block diagram of single phase controller.

The ramping board circuit is shown in figure 3 . The 20 $\mathrm{kHz}$ signal is produced by the 555 timer IC 3 , it's output is amplified by the emitter follower Q1 to provide adequate drive power for the trigger. $C 7$ is a power up reset capacitor to prevent unwanted SCR triggering when the power is turned on but the control is not yet established. The ramp voltage of -10 to $+11 \mathrm{~V}$ is generated by charging up $\mathrm{C} 10$ slowly with negative pulses produced by IC4 and Q2 and is available at the emitter follower output of Q4. The ramp slope can be adjusted by varying the value of resistor PT1. Diode D7 is to prevent the capacitor C10 from discharging when Q2 is off. D9 discharges $\mathrm{C} 10$ when control power is off. This ensures that the ramping voltage will start from -10 Volts every time the power supply is turned on.

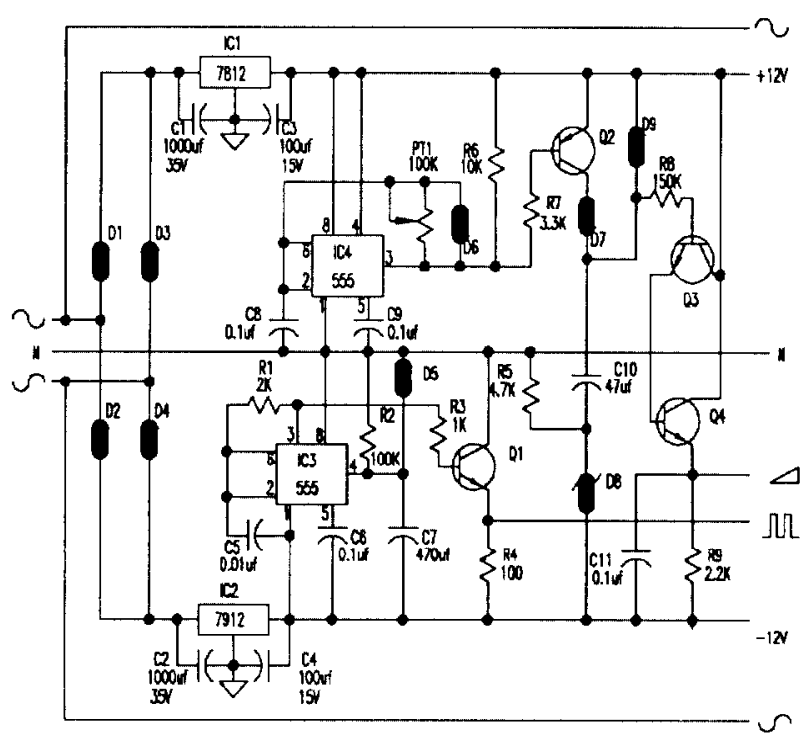

Figure 3. Schematic of ramping board.

The firing circuit, shown in figure 4, provides trigger signal for all the four SCR's. The output of the integrator IC1, which is 90 degrees leading from the sample input of phase $A$, is compared with the ramp voltage. For the positive half cycle of the input phase, when the output of IC1 is lower than the ramp voltage, the output of IC3 will go high enabling Q1 to amplify the $20 \mathrm{kHz}$ signal. This signal is rectified by D9 as the trigger signal for SCR1. Therefore, as the reference voltage ramps from -10 volts to 11 volts, the conduction angle of SCR1 gradually increases from zero to full conduction. For the negative half cycles of the same phase, IC2, IC4 and Q2 generate the trigger signal for SCR2. Since the third phase is fully blocked during this period, a little unbalance in trigger signal will not cause large current in one phase to trip off the $\mathrm{AC}$ circuit breaker or to damage the SCR's,

The 20 second time delay for firing SCR3 and SCR4 is obtained from the 12 bit counter IC6. The counter is constantly reset till the output at IC4 goes to a steady high. After 20 seconds of counting, $Q_{M}$ of IC6 goes high, disabling the clock so that $\mathrm{Q}_{\mathrm{M}}$ remains high. $\mathrm{Q} 4$ is then enabled and amplifies the $20 \mathrm{KHz}$ signal to provide the firing voltage for SCR.3 and SCR4.

\section{SERIES RESISTOR CIRCUIT}

This type of filament power supply evolved as a standby emergency solution when the old 3 phase 3 SCR controlled power supplies failed. This configuration is shown in figure 5 where three $16 \mathrm{ohm}$ resistors are provided by cooking range elements, and are connected in series with the primary of the transformer to limit the initial surge current as the 480 Volts ac source is turned on. 


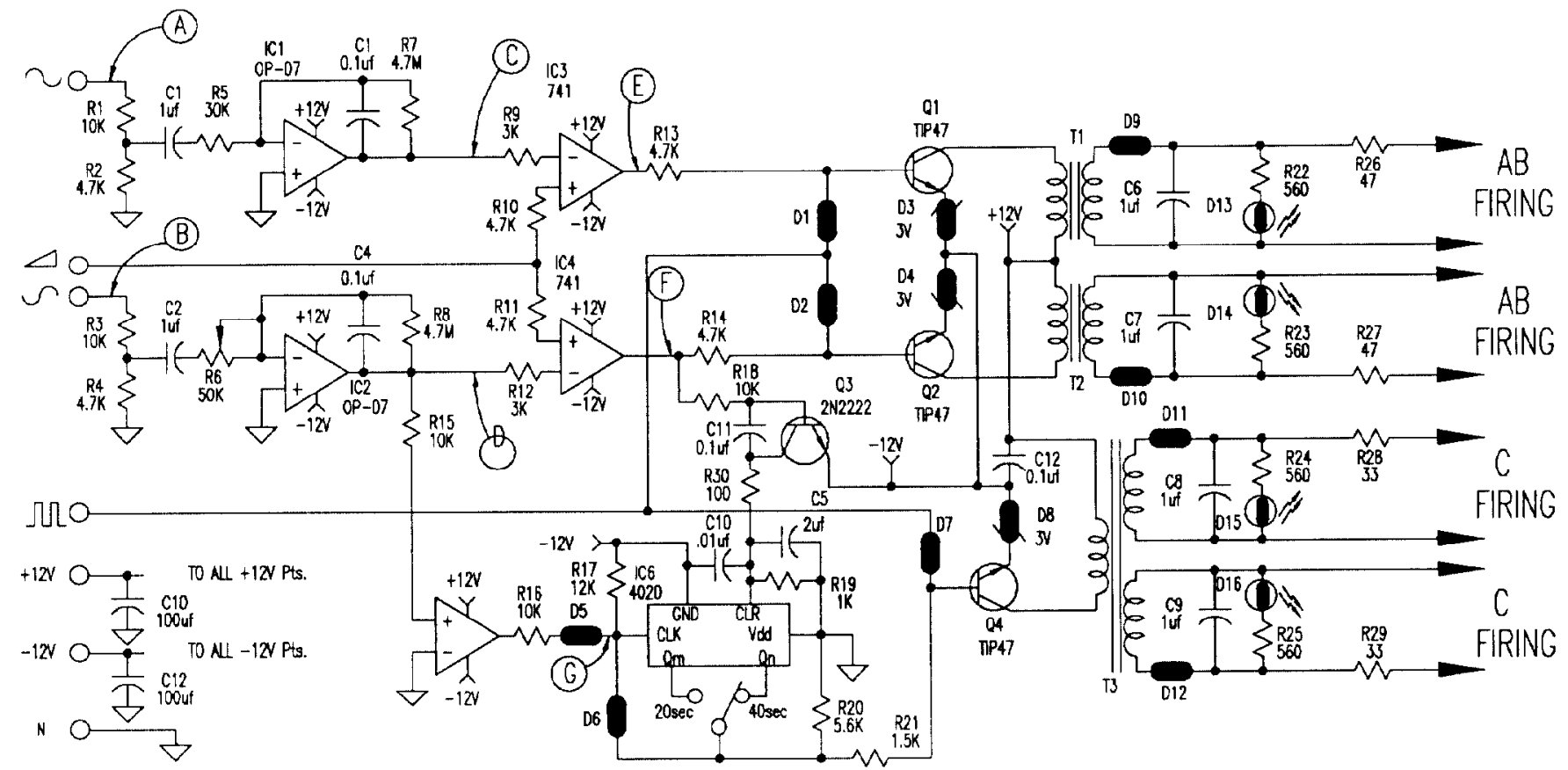

Figure 4. Schematic of firing board.

A time delay relay provides a delay of 20 seconds to allow the filament to reach about $80 \%$ of its resistance and the series resistors are then bypassed. In case of power bump or loss of mains, the time delay relay restarts. The resistors are mounted in a box outside the filament power supply housing and provision is made such that after the filament has reached it's rated voltage and current, the resistor box can be disconnected from the power supply and used for another supply.

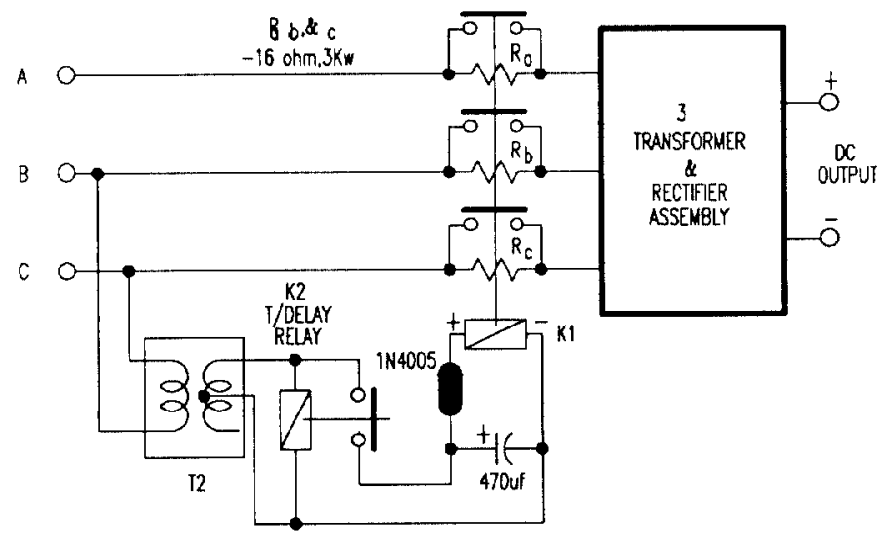

Figure 5. Schematic of series resistor circuit.

\section{CONCLUSIONS}

The newly developed single phase controller has worked for a pcriod of six months without any failure. $\Lambda t$ present all the eight filament power supplies are being modified with this new type of soft start circuit.

\section{ACKNOWLEDGMENTS}

The authors wish to thank Roger Poirier for encouraging the development of such power supplies. Thanks are also to Glen Blaker, Marek Lipnicki, Richard Shanks, Bruce Whiteside, Joseph Holek and Gus Mannes for their help in assembly, testing and troubleshooting the power supplies..

\section{REFERENCES}

[1] K.L. Erdman et al, "TRIUMF amplifier and resonator system," Proceedings of the 6th International Conference, AIPCP 9, 451(1972).

[2] R.H.M Grummer, R.L. Poirier and M.Zach," TRIUMF if system - initial operating problems and their solutions," Proceedings of the 7 th Conference on Cyclotrons and their applications, pp. 167-170(1975). 